\title{
L'invention rhétorique dans la Rhetorique françoise faicte particulierement pour le roy Henri III de Germain Forget
}

JOHN NASSICHUK

The University of Western Ontario

This article examines the place given to the part of rhetoric known as "invention" in the Rhetorique françoise faicte particulierement pour le roy Henry III, a treatise which has been attributed to the jurist Germain Forget. First, this study underlines the relative importance, in Forget's treatise, of this category which traditionally occupies the first place in the ordered disposition of ancient rhetorical treatises. In comparison, reference is made to two other contemporary treatises. Then, second, the analysis shows that the author favours a general idea of invention setting it apart from the technical considerations pertaining to specific theories of "causes". Finally, the third part of the study proposes the hypothesis that this general idea of invention, inspired by Quintilian, bears the stamp of contemporary Humanism, in particular that of the adherents, in the tradition of Erasmus and Politian, to the theory commonly known as "innutrition". At a time when the last of the Valois kings ardently wished to become a father, even commanding that public prayers be recited to this effect, the theory of rhetorical invention rests, perhaps appropriately, on humanist ideas concerning pedagogy.

$\mathrm{D}$ ans son ouvrage important sur l'évolution de la rhétorique ramiste, Kees Meerhoff note que Pierre de la Ramée affirmait, dès l'un de ses premiers écrits, que la rhétorique et la dialectique «sont des sœurs destinées à une conjonction naturelle ${ }^{1} \gg$. Or, les textes qui présentent et développent son enseignement n'apportent que bien peu d'éclaircissements à cette première intuition, si bien que « tout porte à croire qu'il s'est occupé davantage de la séparation théorique des artes que de leur conjonction $^{2} \gg$. En effet, la séparation est renforcée dans la méthode rhétorique des ramistes par l'absence formelle de tout traitement systématique de l'invention. Il est utile de rappeler que l'énumération traditionnelle des cinq parties de la rhétorique situe l'invention à la première place, au sein d'un ensemble dont l'ordre procède de la matière à la présentation, ou du « fond $\gg$ à la « forme $\gg$ :

Les parties d'autre part sont celles que la plupart des maîtres ont indiquées : l'invention, la disposition, le style, la mémoire et l'action oratoire. L'invention (inventio) consiste à trouver (excogitatio) les arguments vrais ou vraisemblables, propres à

Renaissance and Reformation / Renaissance et Réforme 31.4, Fall/automne 2008 
rendre notre cause convaincante. La disposition (dispositio) consiste à mettre en ordre les arguments que l'on a trouvés (rerum inventarum). Le style (elocutio) adapte à ce que l'invention (ad inventionem) fournit des mots et des phrases appropriés. La mémoire (memoria) consiste à bien retenir les idées et les mots. L'action oratoire (actio) consiste à discipliner la voix et les gestes du corps en fonction de la valeur des idées et des mots 3 .

Cette description des parties de la rhétorique montre que l'invention, source de matière véritable ou vraisemblable (rerum verarum aut veri similium), constitue le lieu le plus « naturel »d'une « conjonction » des procédés de la philosophie et de l'éloquence, c'est-à-dire de la dialectique et de la rhétorique ${ }^{4}$. L'invention y apparaît fondamentale en ce sens qu'elle entretient des liens nécessaires avec d'autres parties de la rhétorique, leur fournissant la matière même de leurs expressions finement distillées. C'est pourquoi la mise à l'écart de l'invention dans les traités ramistes est si frappante, comme Alex Gordon l'a bien souligné dans la brève étude qu' il consacre au traité attribué à Germain Forget ${ }^{5}$. En assignant au domaine de la dialectique l'invention de la matière argumentative, la pensée ramiste délimite radicalement le territoire propre de l'éloquence. Alex Gordon déclare en outre, à propos de Forget, que « l'importance extraordinaire qu'il attache au style, aux dépens semble-t-il du contenu, fait de lui un ramiste dans l'esprit, sinon à la lettre ${ }^{6} \gg$.

La présente étude examinera le statut de l'invention dans le traité qui porte le titre Rhetorique françoise faicte particulierement pour le roy Henri III, afin de nuancer cette description du « ramisme » de Germain Forget 7 . Tout d'abord, l'analyse comparera le statut de l'invention dans ce traité à celui qu'elle revêt dans les deux manuels de rhétorique les plus proches en termes chronologiques, adressés au même destinataire royal : l'Avant-Discours de rhetorique ou traicté de l'eloquence de Jacques Davy Du Perron ${ }^{8}$ et le Projet de l'eloquence royale de Jacques Amyot ${ }^{9}$. Elle étudiera ensuite l'idée de l'invention chez Forget en la comparant à celle que l'on trouve chez d'autres théoriciens du XVI ${ }^{\mathrm{e}}$ siècle comme Fabri, Sébillet, Peletier du Mans et Ronsard. Cette étape de l'analyse doit montrer que les propos de Forget sur l'invention correspondent de près, sur plusieurs points, à ceux qui apparaissaient déjà dans les traités de la première moitié du siècle et pendant le règne de Henri II. Enfin, la troisième et dernière partie de l'analyse avancera une hypothèse concernant la particularité de l'invention dans le traité de Forget. Il s'agit de montrer notamment que Forget demeure tributaire d'une tradition humaniste, très prononcée à partir du Quattrocento, dans laquelle convergent les deux visées, distinctes, des manuels de rhétorique et des traités d'éducation ${ }^{10}$. Sans adhérer rigoureusement à une « doctrine » rhétorique héritée des écrits de Pierre de la Ramée, Forget met 
à contribution le traitement ramiste du style dans la longue partie intitulée « de l'élocution ». Au lieu d'éliminer entièrement la catégorie de l'invention, comme le font les traités ramistes, il propose une description détaillée de cette catégorie fondamentale. Sa description souligne l'importance de la préparation intellectuelle comme fondement essentiel de l'art de l'éloquence.

Claude La Charité montre bien, dans son article, que des trois traités de rhétorique destinés au roi Henri III, le texte fragmentaire de Germain Forget est celui qui montre l'affinité la plus grande avec la doctrine ramiste dans le domaine de l'élocution ${ }^{11}$. Il reste à montrer que le rôle assigné à cette réflexion technique demeure circonscrit par la mission pédagogique du traité destiné au roi, car Forget ne décrit pas l'invention comme la science formelle des lieux d'argumentation, mais comme le fruit d'un travail d'apprentissage. Le fait que ce travail commence selon lui dans un cursus d'instruction et de lectures guidées, révèle la priorité qu'il accorde à une culture de l'esprit qui réside d'abord dans la fréquentation d'une riche diversité de textes. Lorsqu'il présente au lecteur les principes du style, il suit de près les définitions des rhétoriques ramistes, qui sont présentées avec une rigueur et une clarté souvent absentes des manuels de l'Antiquité. Mais cette allégeance demeure circonscrite par un aperçu de l'invention qui rapproche Forget d'autres sources et modèles. Son traitement du thème suggère une certaine familiarité avec les traités de l'Antiquité latine, les arts de rhétorique et de poétique de la Renaissance qui privilégient le principe de l'innutrition, les écrits parénétiques adressés aux derniers rois Valois et, dans la même veine, les traités de pédagogie qui insistent sur l'importance d'une grande variété de lectures.

\section{L'invention dans les trois traités principaux}

Des trois principaux traités de rhétorique écrits en français durant les premières années du règne de Henri III, celui attribué à Germain Forget est le seul qui traite explicitement de l'invention comme partie de la rhétorique. Jacques Davy Du Perron et Jacques Amyot tiennent tous les deux un discours général sur les nombreuses qualités de l'éloquence et sur son utilité pour le gouvernement d'un royaume ou d'une république. Tout élément conspire à mieux conseiller le roi, à lui apporter un soutien personnel, dans ces écrits taillés spécifiquement à sa personne et à sa situation politi$q \mathrm{e}^{12}$. Du Perron propose un texte sans divisions catégorielles, une sorte de discours parénétique sur le bon usage, et les atouts suprêmes, de l'éloquence, dans lequel les conseils édifiants se mêlent étroitement aux renseignements techniques. Jacques Amyot présente au roi un texte plus rigoureusement organisé, mais l'identification 
même des chapitres y reflète autant les intérêts particuliers de l'éloquence royale que les conventions établies de la rhétorique. Les propos de ces deux auteurs abordent parfois, il est vrai, l'invention oratoire. Leurs remarques sur ce thème demeurent toutefois éparses et rapides, sans jamais avancer de position précise.

Dans l'Avant-Discours de rhetorique, Du Perron suit Cicéron en soulignant l'extrême difficulté de la tâche de qui cherche à, selon son expression, « rendre un homme parfaitement éloquent $t^{13} \gg$. Cet emploi du verbe « rendre » suggère sans doute quelque souvenir des traités anciens qui présentent le défi du point de vue du maître qui forme un élève. Aussi Du Perron explique-t-il que la première étape de la formation du rhéteur est celle de l'éducation générale :

Premierement il faut faire un fonds et un magasin de toutes sortes de sciences, et acquerir la cognoissance d'infinis sujets, sans laquelle l'abondance des paroles n'est rien qu'un flux de langage inutile et ridicule, car il faut que la vraye eloquence naisse et fleurisse de la cognoissance des choses, comme de sa plante et de sa racine ${ }^{14}$.

Cette description du fonds de savoir nécessaire (« il faut ») à la formation de l'orateur, correspond entièrement à l'idéal de l'éloquence décrit par Cicéron et Quintilien. Selon cette conception ancienne, former le rhéteur idéal est impossible précisément en raison de la variété et de la profondeur des matières que l'homme parfaitement éloquent doit maîtriser. Peu concerné par les aspects techniques de l'éloquence, Du Perron retient le sens le plus large du terme « invention » : puiser les mots convenables dans un trésor lexical préparé à travers de nombreuses lectures. Or, l'auteur n'entreprend de tracer aucun éventail de ces « infinis sujets ». Il énonce le principe, qui concerne l'éducation, avant de passer directement à la seconde étape de son programme, qui correspond de près à la technique de l'élocution ${ }^{15}$. Son traité ne présente donc aucune réflexion soutenue sur la partie première et fondamentale de l'art rhétorique.

Le Projet de l'eloquence royale de Jacques Amyot est plus rigoureusement organisé. Il se divise en quinze parties, ou « chapitres », dont le dernier tiers présente une succession de matières semblable à celle des cinq parties de la rhétorique. Sans faire mention de l'invention comme étant l'une de ces parties, il consacre un bref chapitre à ce qui semble en constituer l'équivalent. Ainsi, le chapitre $\mathrm{X}$ de son traité, intitulé « Des lieux d'où l'on tire les arguments et les passions », se rapproche de la description de l'invention qui apparaît dans certains traités anciens comme la Rhétorique à Herennius. Ici, Amyot égrène les noms d'une série de «lieux » à caractère résolument technique : la définition, le dénombrement des parties, la différence, le genre, les espèces, les mots qui proviennent d'une même source, les contraires, les causes, les effets, les similitudes et les comparaisons. Or, craignant, 
lui aussi, les affres de l'« infini » positif et précis, il déclare que cette procession de noms techniques n'a rien d'une liste exhaustive :

\footnotetext{
J'en laisse toutefois les exemples dont le nombre est infini, explique-t-il, et ne sont les arguments mal aisés à trouver : car comme le chasseur après qu'il a reconnu les gîtes des bêtes sauvages, et environné la forêt de ses toiles, il est impossible qu’y entrant avec ses chiens, et guettant diligemment, il n'en attrape ; aussi quand on a bien remarqué ces lieux, qui sont comme les repaires des arguments, et qu'on les a entourés de la pensée, on ne peut faillir en cherchant d'en rencontrer qui seront propres à la confirmation de notre dire ${ }^{16}$.
}

La métaphore suggère de voir dans la matière de l'invention une source de possibilités vaste et profonde, qui, loin de se laisser maîtriser par les capacités de l'homme, se manifeste surtout à celui-ci par une généreuse abondance. Au lieu de se cacher devant l'esprit de celui qui cherche, cette matière, telle la Nature de l'âge d'or, procède à sa rencontre. Il est évident qu'Amyot, tout en proposant quelques indications techniques à l'apprenti qui souhaite connaître la « méthode » de l'invention, se garde bien d'ouvrir trop large sa réflexion sur le travail d'apprentissage qui nourrit l'inventivité de l'homme éloquent.

Le traitement relativement détaillé que l'auteur de la Rhetorique françoise faicte particulierement pour le roy Henry III accorde au sujet de l'invention le distingue nettement de ses deux contemporains. Forget montre une allégeance à l'ordre classique des parties de la rhétorique, puisque le chapitre intitulé « Del'Invention » constitue la première section de son traité fragmentaire. Ce chapitre est suivi d'un bref paragraphe intitulé « arguments » qui, lui aussi, semble appartenir aux considérations propres à l'invention. Ensuite, la partie majeure de ce document est consacrée aux procédés de l'élocution, ce que la critique a déjà remarqué plusieurs fois. Il est incontestable que l'importance et l'organisation même de cette partie centrale témoigne de l'influence des traités ramistes ${ }^{17}$. Détaillée et riche en citations de poètes contemporains — notamment de Garnier et de Desportes —, elle ressemble en plusieurs endroits à la Rhetorique françoise d'Antoine Fouquelin. Elle est suivie d'une section importante sur l'action, d'un paragraphe sur le « nombre oratoire » et enfin d'une partie intitulée « de l'exorde », interrompue à la fin du document. La disposition des matières reflète ainsi un certain effort de l'auteur pour construire cette première partie de son traité selon le modèle cicéronien des cinq parties de la rhétorique.

Kees Meerhoff affirme avec raison que le traitement des « nombres d'oraison » éloigne Forget de la rhétorique ramiste, puisque « nulle part, il ne rapproche figure de mots et nombre ${ }^{18} \gg$. Il semble donc raisonnable de croire que la matière de cette 
section correspond, non à la réflexion sur le nombre qui apparaît chez Pierre de la Ramée et Omer Talon, mais à celle de la pronuntiatio classique. Il s'agit donc d'une substitution terminologique, ce qui paraît d'autant plus probable que Forget consacre la moitié de ce paragraphe à une réflexion sur les divers effets des sonorités des consonnes ${ }^{19}$. Si l'on admet que la partie réservée au « nombre » constitue en réalité une réflexion sur la prononciation, seule la catégorie de la dispositio demeure absente de la Rhetorique de Forget.

Le traitement de l'invention dans ce traité fragmentaire dépasse largement, quant à la longueur et au détail, celui qui caractérise la plupart des traités d'éloquence, ou même de rhétorique et de poétique, rédigés en français au XVI siècle. Dans la première section de son traité, Forget ne propose pas la métaphore conventionnelle du «trésor » (fonds et magasin) comme le fait Du Perron. Il ne dresse pas, non plus, une liste de procédés techniques propres à l'invention, comme le fait Amyot. De brèves remarques sur ces procédés apparaissent, non dans la section intitulée «De l'invention », mais dans celle qui la suit, plus brève, qui porte sur les « arguments ». C'est ici que l'on retrouve le poncif sur l'infinité de lieux aptes à fournir des arguments à l'orateur ${ }^{20}$. Or, chez Forget, cette remarque sur l'abondance de lieux disponibles à l'invention oratoire découle logiquement de la section précédente, qui traite proprement de l'invention. Dans cette première section en effet, Forget souligne l'importance de la lecture, surtout des auteurs grecs et latins. La phrase conclusive précise que les « raisons et arguments » dont se servent les orateurs dépendent de « ceste partie de l'invention » qui consiste dans l'instruction et dans la « congnoissance des belles choses ». Ainsi, l'organisation de la matière dans les deux premiers chapitres du traité de Forget induit une division claire entre l'invention comme éventail de procédés techniques et celle qui, de toute évidence, intéresse davantage l'auteur : l'invention comme trésor des res et des verba soigneusement accumulés à travers un véritable cursus d'instructions et de renseignements.

Cette idée de l'invention qui procède de la lecture et de l'instruction organisée constitue le fondement essentiel de l'éloquence selon Germain Forget. C'est bien elle qui, dans la perspective de notre auteur, préparera le rhéteur à bien répondre aux exigences sempiternelles de l'art oratoire : « estre instruit en la cognoissance des belles choses, pour en pouvoir discourir plainement en toutes occurrences ${ }^{21} \gg$. La formulation de cet objectif apparemment banal ne laisse pas d'être instructive : « plainement », de manière satisfaisante et même copieuse, en déployant les ressources lexicales du trésor construit grâce à la lecture et au bon enseignement ; « en toutes occurrences », en toute occasion, ex tempore si nécessaire, comme Quintilien le proposait dans le livre conclusif de son Institution oratoire. La mise en place de ce 
trésor lexical et intellectuel constitue l'objet premier du traitement de l'invention dans la Rhetorique françoise faicte particulierement pour le roy Henry III.

\section{L'invention dans l'opuscule de Forget}

Le traitement de l'invention comme catégorie rhétorique a suscité plusieurs remarques de la part de la critique intéressée au traité de Forget. Ces commentaires trahissent parfois une certaine impatience avec le procédé de l'auteur, car si la méthode d'exposition le rapproche des ramistes sur le thème de l'élocution, il ne suit pas pour autant ce modèle quant à l'organisation même de son traité. En effet, la tendance majeure de la critique est de percevoir la Rhetorique de Forget comme un écrit dérivatif du ramisme, en soulignant notamment le fait qu'il privilégie le domaine de l'élocution. Une autre tendance en résulte, qui consiste à diminuer l'importance de l'invention dans le traité. Kees Meerhoff n'hésite pas à affirmer que « l'auteur de la Rhetorique, quel qu'il soit, connaît mal son sujet ${ }^{22} \gg$. Selon ce critique en effet, Forget se trompe de catégories lorsqu'il décrit le principe de l'invention :

[ ... ] ainsi, il dit à propos de l'invention, première des quatre parties de la rhétorique qu'il admet, qu'elle « consiste en choses ou matiere dont l'on doit traicter ou qui servent à icelle. » Il ajoute : «Comme raisons et arguments, elle consiste au chois et elite des dictions et des mots », ce qui se rapporte évidemment à l'élocution plutôt qu'à l'invention ${ }^{23}$.

Vue de cette manière, la discussion même de l'invention ressemble à une simple erreur technique - par une méprise révélatrice, Forget attribuerait à l'invention ce qui appartient en réalité à la catégorie du style. Or, il existe une autre possibilité, tout aussi vraisemblable : que Forget perçoit l'invention non comme un domaine technique analogue à celui de l'élocution, mais comme le vaste tronc du savoir qui réside à la base de l'art rhétorique pris dans son ensemble. Afin de bien mesurer la véracité d'une telle hypothèse, il convient de rallonger un peu la citation de cette partie du traité. Remarquons tout d'abord que l'auteur de la Rhetorique rattache le travail des choix lexicaux à celui qui consiste en l'acquisition de connaissances fondamentales. C'est pourquoi il précise soigneusement les champs de savoir essentiels, qui sont pour lui autant de sources de mots et de choses :

L'invention consiste en choses ou matiere dont l'on doit traicter ou qui servent à icelle. Comme raisons et arguments, elle consiste au chois et elite des dictions et mots. Ceste matière gist en la congnoissance des arts, science, dont l'on peut parler en discours, et principalement de la philosophie morale et de l'histoire ${ }^{24}[\ldots]$ 
La citation rallongée permet de montrer que, pour Forget, la référence « au chois et élite de la diction des mots » demeure étroitement liée à divers champs d'études, véritable source de « raisons et arguments ». Inféodé aux connaissances qui constituent la véritable richesse de l'éloquence, le travail du style découle toujours des trésors de l'invention. Tout porte à croire que Forget ne confond aucunement les catégories de l'invention et de l'élocution. Il cherche plutôt à décrire l'invention d'une manière qui rende compte de la largeur du champ de connaissances qu'elle renferme.

Forget n'est guère seul, au XVI ${ }^{\mathrm{e}}$ siècle, dans cette tentative. Pour le montrer, il suffit de rapprocher sa définition de l'invention de celle qui apparaît dans un écrit français de l'époque de la Pléiade, l’Art poétique de Jacques Peletier du Mans. Au chapitre I, iv de son traité, consacré au sujet « de la Composition du Poème en général : et de l'Invention, Disposition et Elocution », Peletier du Mans propose tout d'abord la définition suivante :

Toutes sortes d'Écrits s'accomplissent de trois parties principales, qui sont Invention, Disposition, Élocution. Invention est un dessein provenant de l'imagination de l'entendement, pour parvenir à notre fin. Elle est répandue par tout le Poème, comme le sang par le corps de l'animal : de sorte qu'elle se peut appeler la vie ou l'âme du Poème ${ }^{25}$.

Comme le premier objet de Peletier est bien la poétique et non la rhétorique, il n'hésite pas à traiter de l'invention, de la disposition et de l'élocution dans un même paragraphe. Cette perspective de poète, plus générale que ne l'est celle des spécialistes de la rhétorique, lui permet de souligner l'entrelacement essentiel des trois aspects majeurs de l'éloquence oratoire. Quelques lignes plus loin, il ajoute à cette première définition une remarque supplémentaire : «Car l’Invention est si digne, que même il y a invention à disposer : et y a invention encore en l'Elocution, savoir est en l'élection des mots ${ }^{26}$. $\gg$ Cette comparaison montre que le traitement de l'invention chez Forget ressemble autant à celui de Peletier qu' à sa résorption par les procédés de l'élocution chez Ramus, Talon et Fouquelin. Un autre rhétoricien de l'époque de Peletier, Pierre de Courcelles, souligne, lui aussi, le lien fort entre l'élocution et l'invention : «Elocution est une appropriation des dictions, et sentences propres et idoynes à l'invention, c'est-à-dire es choses inventees ${ }^{27}$. » Tout suggère en effet que Forget, comme Peletier, cherche à décrire le rapport étroit qui subsiste entre l'invention éloquente et les domaines de savoir, vastes et nombreux, qui la nourrissent. En lui accordant la première place dans les procédés de l'éloquence, il affiche son allégeance à une tradition dont Peletier fut également tributaire. 
La situation traditionnelle de l'invention au début des listes des cinq parties de la rhétorique se manifeste en langue vulgaire dès les premières décennies du $\mathrm{XVI}^{\mathrm{e}}$ siècle, dans Le grant et vray art de pleine Rhetorique de Pierre Fabri ${ }^{28}$. Or, le traitement qu'accorde Fabri au thème de l'invention est nettement moins détaillé que ne l'est celui de Forget, malgré son insistance sur le statut de l'invention comme « principale condition » de la bonne éloquence :

Combien que plusieurs conditions soient requises a ung facteur, la principalle c'est invention, car sans invention subtille, plaisante et nouvelle, le facteur ne sçaura deduire sa matiere plaisante ou utille, et est principalle et necessaire pour trouver ses raisons, prouver ses faitz, pour suader et dissuader. Et après les matieres trouvées, il fault disposer par ordre, mettre les foibles raisons au milieu, les fortes au commencement, et les tresfortes à la fin ${ }^{29}$.

Il est aisé de constater que Pierre Fabri, dans la dernière phrase ici citée, semble mélanger quelque peu les catégories de l'invention et de la disposition. De toute évidence, les théoriciens présupposent l'interconnexion des divers aspects de la rhétorique, comme Pierre Ronsard le confirmera dans l'Abrégé de l'Art poétique français, en déclarant « que la disposition suit l'invention mère de toutes choses, comme l'ombre fait le $\operatorname{corps}^{30} \gg$. La pertinence essentielle de l'invention à toutes les autres parties de la rhétorique constitue, on le voit, un véritable principe que les théoriciens du XVI ${ }^{\mathrm{e}}$ siècle répètent souvent. Il n'est donc pas surprenant que Forget, comme Peletier du Mans et d'autres, s'en serve à son tour, dans un traité qui vise tout d'abord à montrer au jeune roi l'importance d'une bonne base de connaissances générales pour qui souhaite se perfectionner dans l'art de bien parler ${ }^{31}$.

La recommandation de lectures diverses apparaît souvent dans les traités de rhétorique anciens et modernes. Quintilien, on le sait, préconisait une variété de lectures dans plusieurs domaines. Ce même topique se manifeste régulièrement au $\mathrm{XVI}^{\mathrm{e}}$ siècle, dans les traités de rhétorique et de poétique rédigés en français. C'est ainsi que Thomas Sébillet, à titre d'exemple, suggère qu'une partie de l'invention relève de l'art, et une autre des dons naturels du poète ou du rhéteur. Pour ce qui est de l'art, le poète apprenti peut consulter les traités de rhétorique :

Le surplus de l'invention qui consiste en l'art, apprendra le poète des Philosophes et Rhéteurs qui en ont écrit livres propres et particuliers, comme celui que j'ai devant supposé savant en l'art de Rhétorique ${ }^{32}$.

Sébillet rappelle ensuite l'importance des lectures diverses, grâce auxquelles « l'invention, et le jugement compris sous elle se confirment et enrichissent $\gg$. On trouve chez Germain Forget le même renvoi aux traités de rhétorique, à ceci près qu'il souligne la difficulté de ces écrits ${ }^{33}$. L'auteur de la Rhetorique françoise réduit en effet 
le traitement technique de l'invention à un renvoi savant aux ouvrages des rhéteurs anciens. C'est bien dans ce sens qu'il convient de lire ses remarques à propos des divers « lieus », dans la section intitulée « Arguments », qui suit immédiatement celle sur l'invention :

Les lieus dont se tirent les arguments sont infinis et innumerables. Combien que Aristotele, Ciceron, en leurs topiques, et Quintilien au sept de son Institution oratoire, se soint efforcés d'en deduire et amasser un grand nombre, et fauldroit pour les deduire et raconter emplement, les esclaircir et expliquer par exemples, comme ils ont faict, un thome entier et separé ; aussy semblent ils apartenir plus particulierement à un Dialecticien que non pas à un orateur, et suffira les faire remarquer par les lecteurs de sa D. Majesté, et à mesure qu'ils se rencontreront en la lecture des bons autheurs, ou dedans les harangues que luy et sa D. Majesté feront par exercitations pour se dresser et façonner à l'eloquence ${ }^{34}$.

Ces lignes permettent d'entrevoir quelles limites Germain Forget impose à son traitement de l'invention. Il lui semble inutile d'adresser au roi des remarques extensives sur la complexité technique des « lieus »d'argumentation, préférant le renvoyer à des textes spécialisés. Lorsqu'il déclare que ces considérations techniques appartiennent « plus particulièrement à un Dialecticien que non pas à un orateur », la suggestion évidente est que la complexité inhérente à la matière dépasse la visée d'un traité d'introduction générale.

Cette réduction de la part accordée aux préceptes techniques de l'invention n'équivaut pas pour autant à une évacuation de la première catégorie de la rhétorique. Il s'agit pour Forget de réorienter son propos sur l'invention selon les exigences de sa tâche de pédagogue. C'est pourquoi il énumère, non les arcanes spécialisés des lieux d'invention topiques, mais des domaines de lecture utiles à ceux qui souhaitent se former l'esprit à l'art de bien parler. Après la philosophie et l'histoire, Forget conseille à son élève royal l'étude de la « Cronologie », « c'est-à-dire l'observation du tens auquel chacque [histoire] aura esté faicte », et de la Géographie, « c'est-àdire description des lieus ou les choses dont l'on parle se sont passées, car ce sont les vrays, naturels et inseparables compagnes de l'hystoire, sans lesquels elle demeure confuse et destituée de sa lumiere d'illustration $\gg$. Il lui conseille également la lecture des fables anciennes, « pour la ressemblance qu'elles ont à la vérité, tant pour l'intelligence des poëtes que pour celuy qui faict profession de parler ou descrire eslegamment $\gg$. Ici de nouveau, l'auteur se montre soucieux de la charge qu'il impose au roi studieux. Pour ne pas trop l'encombrer avec un programme d'érudition trop austère, le maître suggère un moyen de faire converger les domaines dans la seule lecture des Offices de Cicéron ${ }^{35}$. Un complément utile à cette première lecture, 
« pour le regard de la Géographie et Cronologie » serait Pomponius Mela, « qui en a escrit fort compendieusement ${ }^{36} \gg$. Ces exemples montrent que le premier objectif du traitement de l'invention dans la Rhetorique françoise faicte particulierement pour le Roy Henri III réside dans la préparation adéquate d'un trésor de connaissances apte à nourrir l'éloquence de l'orateur apprenti. Voilà donc le fondement essentiel qui doit permettre au dernier monarque Valois de parler avec aisance « en toutes occurrences $\gg$. Il s'en suit que l'auteur du traité s'intéresse au thème de l'invention, moins pour ses divers aspects formels que comme principe pédagogique de l'«innutrition $\gg$ à travers la lecture ${ }^{37}$.

\section{Le traité pédagogique}

Comment donc caractériser la catégorie de l'invention dans ce traité curieux et fragmentaire ? Tout d'abord, il semble raisonnable de souligner le fait que Forget, plus que ses contemporains, cherche à rendre compte de son fondement culturel et littéraire. Il s'agit pour lui de décrire ce que l'on peut appeler le défi épistémologique de l'invention. Alors que Du Perron et Amyot se contentent de déclarer « infinis $\gg$ les lieux de l'argumentation et de l'éloquence, Forget entreprend d'organiser la matière qui doit nourrir le traitement de ces lieux chez un rhéteur exercé. Sa description de cette matière ressemble aussi aux listes de lecture qui apparaissent dans les traités pédagogiques du Quattrocento, surtout à partir de la redécouverte du manuscrit de l'Institution oratoire ${ }^{38}$. Ces énumérations d'auteurs antiques (et modernes) constituent en elles-mêmes un véritable topos, attesté dans les traités médiévaux déjà, mais auquel les humanistes tributaires de Quintilien, et du De Oratore de Cicéron ${ }^{39}$, accordent une importance particulière. Leur œuvre met en évidence la forte complicité qui lie deux lignes de réflexion : sur la méthode pédagogique et sur les fondements de la rhétorique.

Cette pratique de l'énumération se manifeste également dans le traité de Forget, lorsqu'il se réfère aux divers domaines dont la matière est utile à la formation d'un orateur. Dans les premiers paragraphes consacrés au sujet de l'invention, l'auteur décrit les disciplines de la philosophie et de l'histoire :

La congnoissance de la philosophie qui enseigne les meurs s'acquiert par une lecture methodique des bons auteurs qui sont traictés et enseignés en leurs labeurs, comme Platon, Aristotele, Plutarque, Cicéron, Sénèque, Pline et autres.

L'histoire se doit apprendre semblablement par la lecture des bons autheurs qui sont plus dignes de foy et ont gaingné credit envers les hommes d'estre plus veritables et dignes rechercheurs des antiquités concernant leur subject. S’acquiert aussi par 
la conference ordinaire d'un homme versé en icelles, qui conjoindra aus histoires la Cronologie $\mathrm{e}^{\mathrm{0}}[\ldots]$

Le souci de la méthode de l'apprentissage, et de la mise en place d'un curriculum bien choisi, se manifeste ici de manière évidente. Un tel raisonnement rapproche l'auteur de ceux qui réfléchissent aux méthodes de la pédagogie et de l'étude. D’un intérêt tout particulier à cet égard sont les recommandations concernant le domaine de l'histoire. Ce serait une erreur d'y voir seulement un procédé conventionnel, qui reproduit quelque poncif hérité de l'Antiquité. La revendication de l'importance de l'histoire reflète plutôt une tendance majeure des traités pédagogiques de la Renaissance, dont l'essor remonte au célèbre plaidoyer en faveur de ce genre de lecture formative dans le De Ingenuibus Moribus, texte humaniste souvent réédité au cours $\mathrm{du} \mathrm{XVI}{ }^{\mathrm{e}}$ siècle. Elle reflète aussi sans doute, de façon complémentaire, le raffinement continuel de la théorie et de la pratique de l'historiographie en France, comme les travaux de George Huppert et Claude-Gilbert Dubois l'ont bien montré ${ }^{4}$.

Remarquons en outre que l'auteur ne se contente pas d'énumérer les bonnes lectures. À la vérité, il envisage une situation pédagogique très élaborée, dans laquelle le roi suivra une véritable formation sous le regard attentif d'un maître. Lorsqu'il suggère à Henri III la lecture du De officiis, Forget décrit un exercice de lecture guidée, voire même d'imitation scolaire :

Quelque docte personnage traduira les offices de Cicéron en françois et en fera lecture d'un chapitre pour chacun jour, puis l'explicra à Votre Majesté, discourant sur le subject d'iceluy, raportant tout ce que les autheurs Grecs et Romains en auront escript, plus l'éclaircira des exemples de l'antiquité tiré des histoires et fables anciennes. Votre divine Majesté fera semblable discours apres redigé par escrip, ou elle observera toutes les prescriptions et loys de Rhetorique, et le pourra reciter par cœur devant son homme lettré qui en donnera son advis ; et par inssy sera le moyen d'apprendre, par maniere d'esbat et d'exercice, la philosophie, l'hystoire, les fables conjointes avec l'eloquence, et reduire le tout en pratique ${ }^{42}$.

Le texte de Cicéron devient ainsi non seulement une matière de lecture, mais aussi un objet d'étude, de commentaire et de pratique. Il convient de remarquer également l'insistance sur l'apprentissage «par maniere d'esbat », technique héritée du premier livre de l'Institution oratoire, dans lequel Quintilien traite de l'éducation des jeunes enfants auxquels il faut parfois enseigner par moyen de jeux et de concours amusants ${ }^{43}$.

Afin de bien couvrir une variété de domaines et de réflexions, le texte de Pomponius Mela recevra le même traitement : une traduction française sera lue à haute voix devant le roi par un savant capable d'ajouter viva voce son propre commen- 
taire érudit qui résume la matière d'autres textes pertinents. De façon méthodique, l'auteur précise les étapes d'une séquence et le genre même de considération qui conviendra à la leçon :

Il sera traduit en françois pour en faire lecture à sa D. Majesté. Ce lecteur raportera les advis des autres géographes, tant enciens que modernes, sur un chacun chapitre. Il expliquera et racontra ce qui c'est jamais faict de memorable en chaque pais, par ce raport des hystoriens et poetes ; si a esté regi et administré par Roys et Monarques, ou bien par autre forme de Republique ; les naturels des peuples, leurs loys, meurs et conditions; quels fleuves, montagnes, plaines et forests 44 [... . ]

Les livres ainsi commentés ne seront pas la seule ressource mise à la disposition de l'élève royal. Toujours dans l'esprit de Quintilien, Forget propose une variété de sources d'apprentissage, afin de favoriser l'efficacité du travail pédagogique. C'est ainsi que l'étude de la géographie, par exemple, exige quelque recours à des représentations visuelles :

$[\ldots]$ et pour en avoir congnoissance plus claire sera une carte universelle tendue en la chambre du cabinet de sa Divine Majesté et oultre icelle aura le dit homme de lettres cartes particulieres sur lesquelles il montrera plainement et comme à l'œil la situation des choses dont il fera recit ${ }^{45}$.

Une telle initiative méthodologique franchit les limites du domaine de l'éloquence et situe le propos de l'auteur sur le terrain de la réflexion pédagogique. La volonté de trouver divers moyens de transmettre le savoir participe du même esprit que la suggestion de divertir l'élève en le faisant apprendre « par maniere d'esbat ». Héritier d'une tradition au sein de laquelle convergent la théorie de la formation de l'orateur et les principes d'éducation d'un jeune noble, l'auteur de la Rhetorique françoise faicte particulièrement pour le roy Henry III révèle sa dette au genre du traité pédagogique lorsqu' il aborde le thème de l'invention. Sa vision de ce grand thème l'amène à chercher les racines, les fondements essentiels du principe. En parlant de l'invention, il laisse de côté délibérément les aspects techniques, ceux notamment des causes et des lieux, car ceux-ci sont propres aux considérations de rhéteurs dont l'éloquence se déploie dans les instances juridiques. Il leur préfère une série de lieux plus généraux, qui correspondent parfaitement aux domaines de formation et surtout de lecture conseillés à l'éducation de jeunes hommes de l'aristocratie destinés à la vie active ${ }^{46}$.

GERMAIN FORGET SE DISTINGUE de ses deux contemporains plus célèbres, Jacques Amyot et Jacques Davy Du Perron, dans au moins deux aspects de sa discussion de 
la rhétorique. Lorsqu'il traite de l'élocution, il déploie une série de définitions qui témoignent de sa dette aux traités de rhétorique associés à l'œuvre et à l'enseignement de Pierre de la Ramée. Mais à la différence de ce modèle important, Forget ne s'astreint pas à une définition bipartite de l'art oratoire. La présence même, au début du traité, d'une section assignée à l'invention comme partie de la rhétorique, l'éloigne déjà en principe des ramistes. Cet écart fondamental s'accroît encore lorsque l'on considère que son traitement de l'invention correspond moins à une science formelle des lieux d'argumentation — semblable en plusieurs points à la dialectique — qu'à une vision globale de la formation de l'esprit. C'est sans doute aussi cette même idée de la rhétorique comme science universelle, qui transparaît dans la description de l'Hercule qu'il fournissait à Madeleine de Savoye : «Lucian recite que Hercule surnommé le Gaulois, trainoit apres luy grand nombre de personnes liées d'une chaine d'or, par la langue : ce que ne faut entendre fabuleusement, ains estimer que cet Hercule signifioit un receptacle plein de toutes sortes de sciences, lequel par le miel distillant de sa bouche, rendoit les ignorans comme tous esmerveillez, d'oyr resonner le torrent de son eloquence ${ }^{47}$. »

\section{Notes}

1. Kees Meerhoff, Rhétorique et poétique au XVIe siècle en France: Du Bellay, Ramus et les autres, Leyde, E. J. Brill, 1986, p. 183.

2. Ibid.

3. Cicéron, De l'Invention, I, vii, $9:$ : partes autem eae quas plerique dixerunt : inventio, dispositio, elocutio, memoria, pronuntiatio. Inventio est excogitatio rerum verarum aut veri similium quae causam probabilem reddant ; dispositio est rerum inventarum in ordinem distributio ; elocutio est idoneorum verborum ad inventionem accommodatio ; memoria est firma animi rerum ac verborum perceptio ; pronuntiatio est ex rerum et verborum dignitate vocis et corporis moderatio » (texte établi et traduit par Guy Achard, Paris, Belles Lettres, collection des Universités de France, 1994, p. 64).

4. Pour une discussion générale du principe de l'invention dans la rhétorique ancienne et à la Renaissance, voir l'ouvrage essentiel de Francis Goyet, Le sublime du «lieu commun ». L'invention rhétorique dans l'Antiquité et à la Renaissance, Paris, Honoré Champion, 1996.

5. Alex Gordon, « Ramist Influence in Germain Forget's Rhétorique françoise faicte particulierement pour le Roy Henry troiziesme (1583) $\gg$, Romance Notes, XXIII, $\mathrm{n}^{\circ}{ }_{3}$, printemps 1983, p. $260:$ : This demotion of the art of content development is of special significance in the XVIth century. Traditional rhetoric had included both the art of argument and the art of style and so had encroached on the territory of dialectic. In mid-century 
this confusion was removed by the well-known reforms of Pierre de la Ramée who assigned the art of argument exclusively to dialectic and limited rhetoric to the art of style and delivery alone. $\gg$

6. Ibid. : « However the extraordinary importance he attaches to style, at the expense it would seem of content, makes of him a Ramist rhetorician in spirit, if not in content. »

7. Mon analyse prend comme texte de base la transcription du manuscrit de Modène, qui apparaît dans Precetti di rettorica scritti per Enrico III re di Francia, édition de Giulio Camus, Modène, Antica Tipografia Soliani, 1887. Référence sera faite aux paragraphes numérotés dans cette édition.

8. Jacques Davy Du Perron, Avant-discours de rhetorique, ou Traitté de l'eloquence [1579], dans Euvres diverses, Paris, Pierre Chaudière 1633, p. 759-770.

9. Jacques Amyot, Projet d'éloquence royale, préface de Philippe-Joseph Salazar, Paris, Les Belles Lettres, coll. « Le corps éloquent », 1992.

10. Sur ce point, je me permets de renvoyer à mon article « L'influence de Quintilien dans les traités pédagogiques du Quattrocento », dans Perrine Galand-Hallyn, Francis Goyet, Fernand Hallyn et Carlos Lévy (sous la dir. de), Quintilien ancien et moderne, Turnhout (Belgique), Brepols, collection «Latinités », à paraître.

11. Voir l'article de Claude La Charité, « Les trois institutions oratoires à l'usage de Henri III, un compendium des traditions latine, hellénistique et humaniste » dans le présent dossier. Je remercie l'auteur de m’avoir communiqué cet article important avant publication.

12. On lit ainsi chez Du Perron une description très circonstanciée de l'utilité propre à la rhétorique dans une monarchie comme celle du dernier Valois. Voir l'Avant-Discours de Rhetorique, p. 760, où il précise d'abord que le domaine de la rhétorique est naturellement moins large dans une monarchie que dans une république, avant d'assigner à l'éloquence une série d'occupations qui intéressent la gouvernance du royaume : «Il y a les ambassades vers les Princes estrangers, il y a les propositions au Conseil d'Estat, il y a les relations, quand on vient rendre compte de quelque longue commission qu'on exerce; il y a les harangues qu'on peut prononcer aux Parlements, aux principaux sieges des Provinces, lors qu'on y prend possession de quelque gouvernement ou de quelque authorité ; il y a celles qu'on peut faire aux corps de villes, et aux assemblées du peuple, et principalement en une saison telle qu'est la nostre, c'est-àdire, durant les guerres civiles, menées par des pretextes populaires, comme pour la Religion. 》

13. Ibid., p. 763 .

14. Ibid., p. 763 .

15. Ibid., p. 763 : « Secondement l'Orateur doit estre riche et abondant de beaux mots, de belles constructions, de belles figures, pour revestir et orner les conceptions de son esprit : par ce qu'encore que les choses tiennent bien le lieu de la principale partie, et soient comme les os, les nerfs, et les muscles de l'oraison, si est-ce qu'elles ont besoin de peau, d'en-bon-point, et de couleur pour leur donner la grace et la beauté. » 
16. Jacques Amyot, Projet d'éloquence royale, ouvr. cité, p. 78-79.

17. Voir Alex Gordon, « Ramist Influence », art. cité, p. 259.

18. Kees Meerhoff, Rhétorique et poétique au XVI siècle, ouvr. cité, p. 258.

19. Il semble qu'ici Forget attribue une terminologie fréquemment rencontrée dans les traités modernes à un principe dont le fonds substantiel remonte aux traités anciens. Voir à titre d'exemple les $\$ 28-29$ : « Nous debvons fuir, tant en vers que en prose, la rencontre de plusieurs mots rudes et pierreus, comme de ceulx qui commencent par ceste lettre R, ou qui en sont composez, et ou se trouve reiteration de mesmes consonnes ; et pareillement fault avoir ceste discretion qu'un mot finissant en voyelles, autres qu'en feminin, rencontre immediatement un autre mot commencent aussy par la voyelle, par ce qu'il s'en faict une mauvaise et facheuse lieson, car les dictions n'ont point un son sollide, ferme et rempli, si les voielles ne sont liées avecques leurs consonnes, comme la chaus avecque le sablon ou siment. Entre les moins virilles dont la rencontre et frequente suitte ait plus mauvaise grace est la lettre $S, T, Z$, pour ce qu'elles font un sifflement en les prononcant qui desagree aus oreilles. »

20. Voir infra, note 35 .

21. Germain Forget, Rhetorique françoise, ouvr. cité, $\mathbb{S}_{4}:$ : Voi la les deus moyens les plus promps et facilles et artificiels que l'on puisse tenir pour V. D. Majesté, pour estre instruit en la congnoissance des belles choses, pour en pouvoir discourir plainement en toutes occurrences, comme sa D. Majesté le sçaura bien juger apres l'avoir essayé. »

22. Kees Meerhoff, Rhétorique et poétique au XVI siècle, ouvr. cité, p. 258.

23. Ibid., p. 258.

24. Germain Forget, Rhetorique françoise, ouvr. cité, $\$ 1$.

25. Jacques Peletier du Mans, Art poétique [1555], dans Traités de poétique et de rhétorique à la Renaissance, édition de Francis Goyet, Paris, Libraire Générale Française, 1990, p. 251-252.

26. Jacques Peletier du Mans, Art poétique, ouvr. cité, p. 252.

27. Pierre de Courcelles, La Rhetorique, Paris, G. Le Noir, 1557, $\mathrm{f}^{\circ} 2, \mathrm{v}^{\mathrm{o}}$.

28. Pierre Fabri, Le grant et vray art de pleine Rhetorique [1521], publié avec introduction, notes et glossaire par Antoine Héron, Genève, Slatkine, 1969 (réimpression de l'édition de Rouen, 1889-1890), p. 15. Voir aussi Pierre de Courcelles, La Rhetorique, ouvr. cité, $\mathrm{f}^{\mathrm{o}} 2, \mathrm{r}^{\mathrm{o}}$ : « Doncques premierement et principalement faut observer à l'Orateur futur, Invention, Disposition, Elocution, Memoire, et Prononciation. »

29. Pierre Fabri, Le grant et vray art de pleine Rhetorique, ouvr. cité, p. 18.

30. Pierre de Ronsard, Abrégé de l'art poétique français [1565], « De l'invention », dans Traités de poétique et de rhétorique de la Renaissance, ouvr. cité, p. 472.

31. Il s'agit là donc d'une tendance générale, dans les arts de rhétorique en France pendant la deuxième moitié du XVI ${ }^{\mathrm{e}}$ siècle. L'importance de l'invention dans ces traités est caractéristique même de certaines œuvres du règne de Henri IV. C'est le cas notamment chez Pierre de Deimier, L’Académie de l'art poétique [ ... ], Paris, Jean de Bordeaulx, 1610 . 
32. Thomas Sébillet, Art poétique français [1548], « De l'invention, première partie de Poésie » dans Traités de poétique et de rhétorique de la Renaissance, ouvr. cité, p. 5859.

33. Germain Forget, Rhetorique françoise, ouvr. cité, $\$_{4}: \ll$ De ceste partie d'invention despandent les raisons et arguments dont les orateurs, et toutes autres personnes qui discourent, se servent et accommodent pour la preuve et soustien de leurs propositions, lesquels arguments sont tirés de certains lieus dont les Rhetoriciens traicttent abondamment et toutefois avec grande obscurité. »

34. Ibid., $\$ 4-5$.

35. La référence à ce traité de Cicéron apparaît également chez Pierre Fabri, dans les premières lignes du prologue à son Grant et vray art de pleine Rhetorique, ouvr. cité, p. 5 : « Tulles, en son premier livre De Officiis, dit que le lyen qui tient les hommes conjoinctz en benigne communité, c'est raison avec oraison ou eloquence, laquelle eloquence conduite de raison enseigne, apprent, communique, dispute, juge entre les hommes et les conjoint en naturelle compaignie. »

36. Germain Forget, Rhetorique françoise, ouvr. cité, $\$_{3}$.

37. Voir, sur ce principe, Perrine Galand-Hallyn, Fernand Hallyn et Jean Lecointe, «L'inspiration poétique au Quattrocento et au XVI ${ }^{\mathrm{e}}$ siècle »; et Perrine GalandHallyn et Luc Deitz, « Poésie et imitation au XVI siècle », dans Perrine GalandHallyn et Fernand Hallyn (sous la dir. de), Poétiques de la Renaissance. Le modèle italien, le monde franco-bourguignon et leur héritage en France au XVI siècle, Genève, Droz, 2001, p. 130-131 et 468-469.

38. Il convient de noter que Germain Forget montre ailleurs une certaine familiarité avec la pensée de Quintilien. Voir ainsi le Panegyrique, à Tresillustre et Tres-vertueuse Dame, Madame Magdaleine de Savoye, Duchesse de Montmorency (Paris, Guillaume Auvray, 1576), dans lequel il fait référence à l'auteur de l'Institution oratoire, $\mathrm{f}^{\circ} 29, \mathrm{r}^{\circ}$ : «Quintilian disputant de l'art et de la nature, combien ces choses sont requises à un Orateur disoit, que le naturel estant seul à grande vertu, vigueur, et force mais l'art dégarni de naturel ne peut rien : estant semblable au navire flottant sur l'eau sans avirons, voile, ny gouvernail, ny Pilotte, et par ce moyen exposé à la misericorde des vents. »

39. Christian Mouchel, Cicéron et Sénèque dans la rhétorique de la Renaissance, Marburg, Hitzroth, 1990, passim et Jean Lecointe, L'Idéal et la différence. La perception de la personnalité littéraire à la Renaissance, Genève, Droz, 1993, p. 210 et suiv.

40. Germain Forget, Rhétorique françoise, ouvr. cité, \$2.

41. George Huppert, L'idée de l'histoire parfaite, traduction française de Paule et Françoise Braudel, Paris, Flammarion, 1972 ; Claude-Gilbert Dubois, La conception de l'histoire en France au XVI $I^{e}$ siècle, Paris, Nizet, 1977.

42. Germain Forget, Rhétorique françoise, ouvr. cité, \$2-3.

43. Quintilien, Institution oratoire, I, iii, 8-13.

44. Germain Forget, Rhetorique françoise, ouvr. cité, \$3-4.

45. Ibid., $\$ 4$. 
46. On peut souligner à ce propos la louange des livres et de l'utilité de la lecture pour la vie publique, qui apparaît dans le panégyrique en prose que Germain Forget adresse à Madeleine de Savoye. Voir ainsi le Panegyrique, à Madame Magdaleine de Savoye, ouvr. cité, fo ${ }^{\circ} 19, \mathrm{r}^{\mathrm{o}}$ : « Vous estes, Madame, deuëment informée de l'antiquité des lettres : comment de tout temps elles ont eu cours és republiques bien policiées : quelles utilitez elles apportent : et combien la lecture d'icelles est necessaire aux Monarques, Roys, Princes, et grands Seigneurs : d'autant que par cela se découvrent les choses que leurs familiers et domestiques ne voudroient, ou paradvanture n'oseroient leur faire sçavoir et entendre : ce sont elles qui ne rougissent de honte, pour dire verité : ce sont elles qui ont jetté les premiers fondements des Monarchies, qui les ont conduittes si brusquement au havre de tranquillité, qui embellissent decorent, illustrent, la voute de cet edifice terrestre. Sans elles les citadins d'une ville ne pourroient vivre en repos dedans leurs propres habitations : ny plus ny moins que le corps n'auroit moyen exercer ses fonctions naturelles l'ame s'estant absentée. Si les armes sont necessaires grandement pour l'amplification d'un Royaume, les livres ne sont moins proffitables pour la conservation d'iceluy. »

47. Germain Forget, Panegyrique à Magdaleine de Savoye, ouvr. cité, $\mathrm{f}^{\circ}{ }_{24}, \mathrm{v}^{\mathrm{o}}$. Quelques lignes plus loin dans le même texte adressé à la veuve d'Anne de Montmorency, Forget avance l'idée que le plaisir de l'éloquence, qui naît de la connaissance des lettres, lie le rhéteur (ou l'auteur) et sa destinataire cultivée, $\mathrm{f}^{\mathrm{o}} 24, \mathrm{v}^{\mathrm{o}}$ à $25, \mathrm{r}^{\mathrm{o}}$ : « Voila doncq' ce que j'ay peu recueillir d'antiquitez touchant l'excellence des lettres : en quoy si je me suis un peu longuement égaré de mon chemin, et forligné de mon propos, je croy que je seray trouvé devant un chacun excusable, tant à raison que je me confie rencontrer des lecteurs patiens : que mesmes pour cause que le long progrez du subject, conduict avec soy quelque Deesse de persuasion, dont Ennius parle : et non sans occasion : car il est bien facile (comme porte l'ancien proverbe) louenger Hercule dedans Lacedemone, et un bourgeois Athenien dedans Athenes : à joindre que les discours precedents, employez à la congratulation des arts litteraires, (qu'aucuns ont nommé liberaux, par ce qu'ils conviennent seulement aux esprits de franche et libre condition) redondent entierement sur la creature raisonnable, qui si bravement en tire l'explication et practique. » 